\title{
PROBABILITY OF EXCEEDING THE CRITICAL SHEAR STRESS FOR SAND MOTION IN SPECIFIC WAVE AND CURRENT CONDITIONS
}

\author{
Pierre-Yves Henry ${ }^{1}$, Alf Tørum², Øivind Arntsen², Dag Myrhaug ${ }^{1}$ and Muk Chen Ong ${ }^{3}$
}

\begin{abstract}
This study is focusing on the threshold of sand motion under random waves combined with a following current. The analysis is based on some flume experiments realized over a natural sand bed for different flow conditions (waves and currents). The main result comes as a map of the probability to exceed the threshold of sand motion, as a function of a wave and a current mobility parameter. These observations are compared to methods predicting the bed shear stress using an equivalent monochromatic wave, and links between the probability of exceeding the critical shear stress for initiation of sand motion and the calculated maximum bed shear stress are found.

Keywords: threshold of motion; random waves; critical shear stress
\end{abstract}

\section{INTRODUCTION}

Erosion and sand transport have been studied for decades and formulae for the sand transport rates as well as the critical shear stresses for sand motion have been developed; to some extent based on experiments. The critical shear stresses may be obtained by the Shields diagram. For random waves, the Shields parameter is frequently based on the significant wave height and spectral peak period; see e.g. Soulsby (1997). A sand/gravel bed may then be considered stable if the Shields parameter calculated from these parameters does not exceed the critical Shields parameter. Obviously the shear stress in a given storm may frequently exceed the critical Shields parameter due to the variation of the irregular waves, although the calculated Shields parameter does not exceed the threshold of sediment motion, based on using the equivalent monochromatic wave. Myrhaug and Holmedal (2011) investigated theoretically the statistical distribution of the bottom shear stresses for different conditions. From these theoretical considerations the probability of exceeding the critical shear stress can be calculated. However, it is also well known that the bed shear stress under waves and current is a result of non-linear interaction. Different approaches have been developed to describe the complexity of these processes (Soulsby 1997, Soulsby and Clarke 2005), often using an equivalent monochromatic wave to represent the random waves.

In addition to the difficulty brought by this non-linear process, the definition of the critical shear stress comes with a considerable scatter as it is based upon experimental data (Soulsby and Whitehouse 1997). Moreover, it is not trivial when it comes to natural sand, as the critical shear stress may vary strongly depending on the mixture of different grain sizes (Wilcock 1993, Shvidchenko et al. 2001).

In order to investigate this, Henry (2011) carried out tests providing results of a simple analysis of experimental data, focused on the threshold of motion of sand particles under random waves plus current. This paper presents the main results, and compares them to existing theories for predicting bed shear stresses under random waves plus current.

\section{LABORATORY EXPERIMENTS}

The initiation of sand motion on a flat sand layer under random waves alone and random waves plus currents has been investigated through a series of laboratory tests. Fig. 1 shows the test set-up in a wave flume.

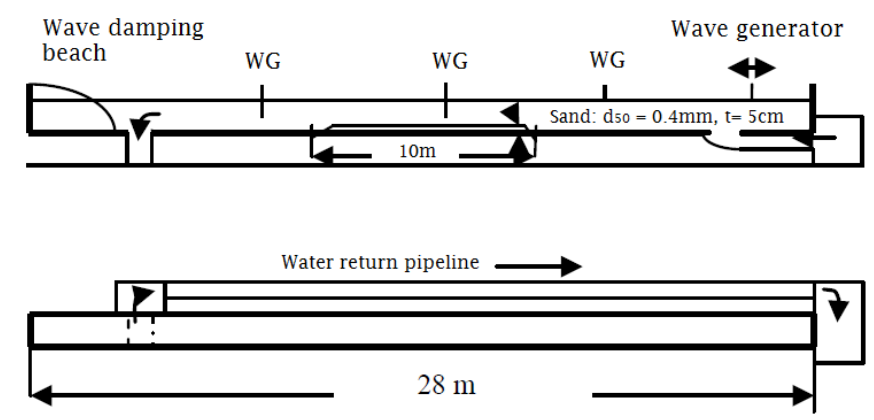

Figure 1 - Wave flume set-up. Flume dimensions: $28 \mathrm{~m}$ long, $0.6 \mathrm{~m}$ wide, $0.5 \mathrm{~m}$ water depth.

\footnotetext{
${ }^{1}$ Department of Marine Technology, Norwegian University of Science and Technology, Trondheim, NO-7491, Norway

${ }^{2}$ Department of Civil and Transport Engineering, Norwegian University of Science and Technology, Trondheim, NO-7491, Norway

${ }^{3}$ Department of Structural Engineering, MARINTEK, Trondheim, NO-7055, Norway
} 
A layer of sediments consisting of sand with median grain size diameter $d_{50}=0.4 \mathrm{~mm}$ was disposed at the bed of the flume $\left(\mathrm{d}_{10}=0.05 \mathrm{~mm}, \mathrm{~d}_{90}=1.3 \mathrm{~mm}\right)$. This layer covered the width of the flume with a thickness of $5 \mathrm{~cm}$ and a length of $10 \mathrm{~m}$. Random waves were generated following a JONSWAP spectrum. For some tests a current in the wave propagation direction was also applied. The different current speeds applied were 0, 10, 20, 30, 35, and $40 \mathrm{~cm} / \mathrm{s}$. For each current condition, three wave periods were tested (1.5, 1.8 and 2s). For a given combination of current-wave period, the wave height has been increased gradually approximately from $4 \mathrm{~cm}$ to $20 \mathrm{~cm}$. A diagram of the conditions tested is given in Fig. 2. The measured data were waves, currents, and visual observations of the sand motion under individual irregular waves.

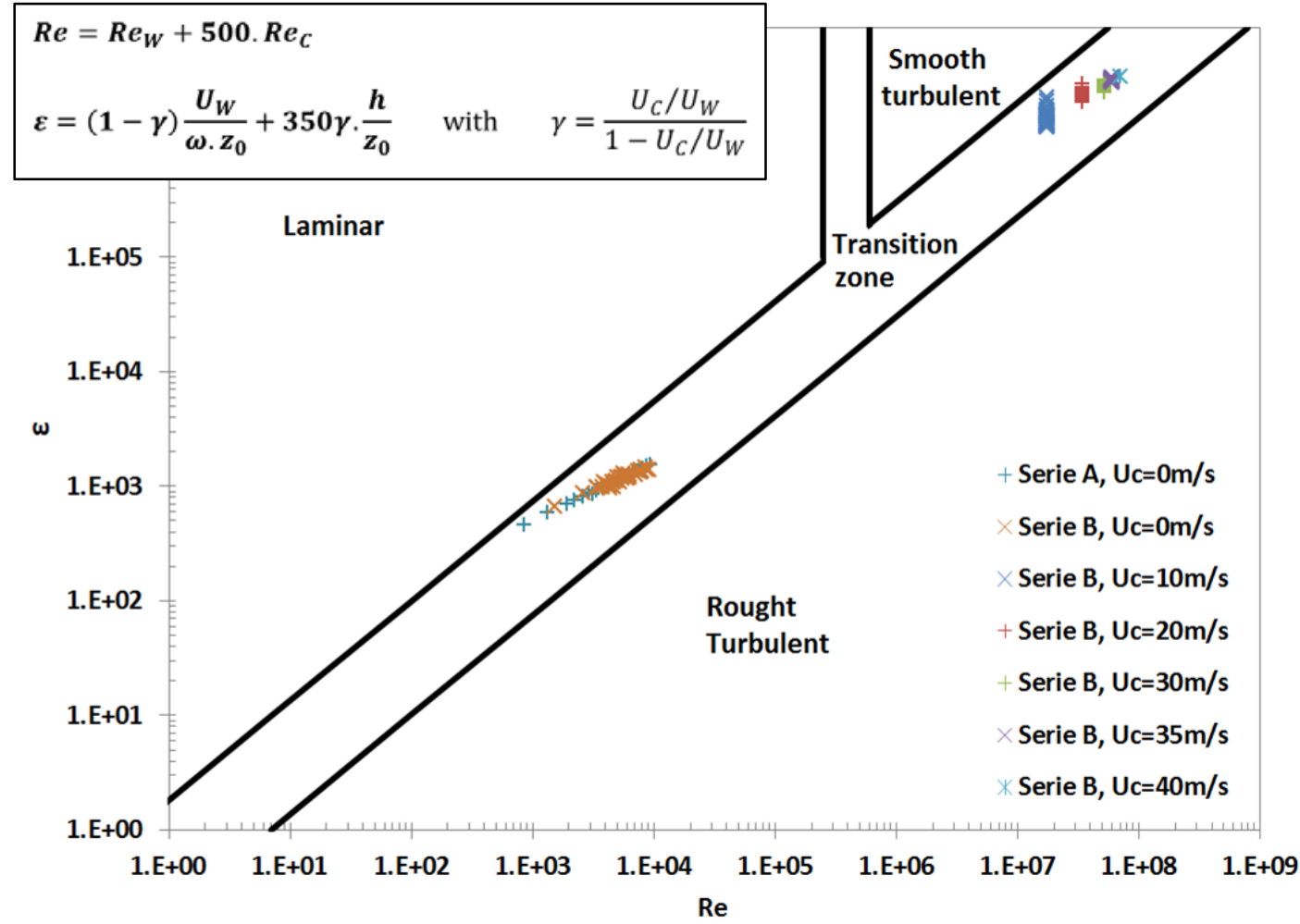

Figure 2 - Diagram of the flow condition tested following the description made by Tanaka and Thu (1994). The Reynolds number (Re), based on the Reynolds numbers for waves alone and current alone $\left(\operatorname{Re}_{w}=U_{w}{ }^{2} / \omega v\right.$ and $R e_{c}=U_{c} h / v$, respectively) is valid for any combined flow. $\varepsilon$ is a roughness parameter depending on the flow characteristics.

As seen in Fig. 2, all the tests are in a transition zone between laminar and rough turbulent for waves alone, and smooth and rough turbulent for combined flows. $U_{C}$ is the mean (during the test period) current velocity in the free stream; $U_{W}$ is the rms (root-mean-square) value of the maximum near-bed orbital velocity obtained from linear wave theory by using the dispersion relationship for waves plus current, the significant wave height $H_{S}$, the spectral peak period $T_{P}$, and the water depth $h . \omega$ is the angular wave frequency associated with $T_{P}, v$ is the kinematic viscosity of the water, and $z_{0}$ is the bed roughness length.

\section{RESULTS AND DISCUSSION}

\section{Stochastic observation of the threshold of motion}

The main result is obtained by using a stochastic description to define the threshold of sand motion, based on the experimental data. This means that for a given test (8min-long run), the number of waves initiating the sand motion under the central wave gauge has been counted. This number divided by the total number of waves gives the observed probability of exceeding the threshold of motion.

This probability has been mapped over a domain characterizing the flow conditions, using the wave and current mobility parameters $\mathrm{Mp}_{\mathrm{W}}$ and $\mathrm{Mp}_{\mathrm{C}}$, respectively:

$$
\begin{aligned}
& M p_{W}=\frac{U_{W}^{2}}{g(s-1) d_{50}} \\
& M p_{C}=\frac{U_{C}^{2}}{g(s-1) d_{50}}
\end{aligned}
$$

where $\mathrm{g}$ is the acceleration due to gravity, $\mathrm{s}=\rho_{\mathrm{S}} / \rho$ with $\rho_{\mathrm{S}}$ and $\rho$ as the density of sand and water, respectively. 
Fig. 3 shows the isocurves of the portion of waves that move the sand grains for a given sea state and current, e.g. the probability of exceeding the critical shear stress for given values of $\mathrm{Mp}_{\mathrm{w}}$ and $\mathrm{Mp}_{\mathrm{C}}$. It appears that for a given wave activity $\left(\mathrm{Mp}_{\mathrm{w}}\right)$, the probability of initiation of sand motion increases as the current $\left(\mathrm{Mp}_{\mathrm{C}}\right.$ $>2$ ) increases; for a given current, the probability of initiation of sand motion increases as the wave activity increases. It should be noted that the curvature of the lines is not necessary physical, but a result of fitting curves to the data by using splines. However, the main trend of the curves is correct. Despite a scatter in the experimental data, the results in Fig. 3 do not depend on any flow regime assumption, and are valid for any combination of waves and currents.

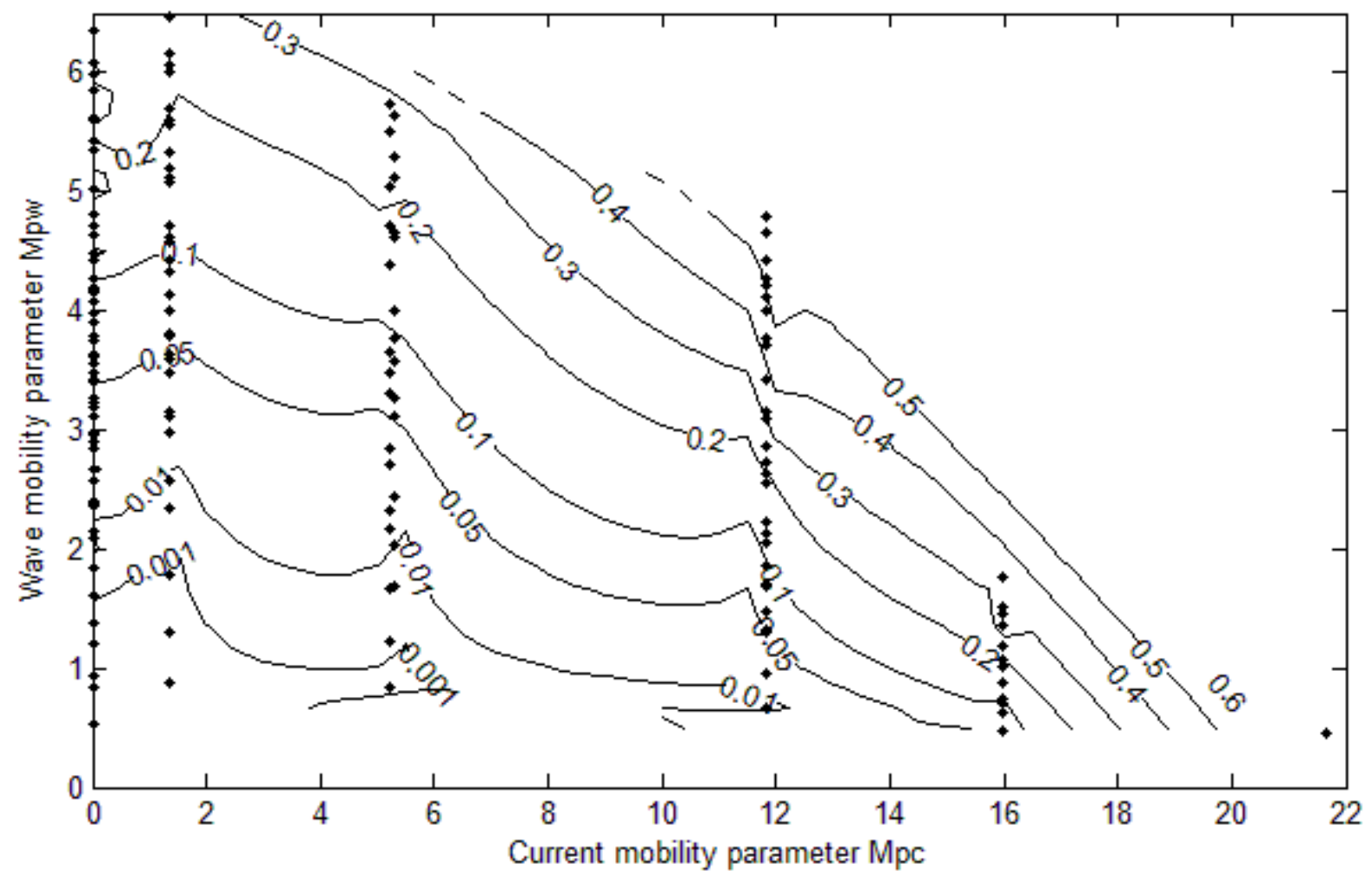

Figure 3 - Isocurves of the probability of initiation of sand motion vs. the wave and current mobility parameters. The points represent the experimental data, and the curves are a result of best fit to the data by using splines.

\section{Critical shear stress and predicted maximum shear stress, Tanaka and Thu (1994)}

The initiation of the sand movement is the result of the maximum bed shear stress exceeding the critical shear stress $\left(\mathrm{T}_{\mathrm{cr}}\right)$. It is then of interest to compare the observed movements of the sand to the calculated maximum shear stress. Here the Tanaka and Thu (1994) model is adopted to calculate this fraction of the bed shear stress, and it is based on using an equivalent monochromatic wave (significant values). The maximum bed shear stress is derived as follows:

with

$$
\tau_{\max }=\rho \frac{f_{c w}}{2} U_{W}^{2}
$$

$$
f_{c w}=f_{2}\left[f_{1} f_{c w(L)}+\left(1-f_{1}\right) f_{c w(S)}\right]+\left(1-f_{2}\right) f_{c w(R)}
$$

where $\mathrm{f}_{\mathrm{cw}(\mathrm{L})}, \mathrm{f}_{\mathrm{cw}(\mathrm{S})}, \mathrm{f}_{\mathrm{cw}(\mathrm{R})}$ are the friction coefficients for the laminar, smooth turbulent and rough turbulent flow regimes, respectively, for combined waves and current, $f_{1}$ and $f_{2}$ are weight coefficients depending on the flow parameters; for more details see Tanaka and Thu (1994). This model is valid for all combined flow regimes, except for waves alone; thus it can be applied in this study for the combined flow situations.

Fig. 4 compares the observed probability to initiate the sand motion with the values of the maximum bed shear stresses, calculated by the Tanaka and Thu (1994) method. These values are calculated for each test case and presented as a function of $\mathrm{Mp}_{\mathrm{c}}$, which explains their location at the same values of $\mathrm{Mp}_{\mathrm{c}}$. It appears that the maximum bed shear stress increases as the wave action increases. From the Shields diagram presented by Soulsby and Whitehouse (1997) (upper figure), two thresholds are considered: the critical shear stress predicted 

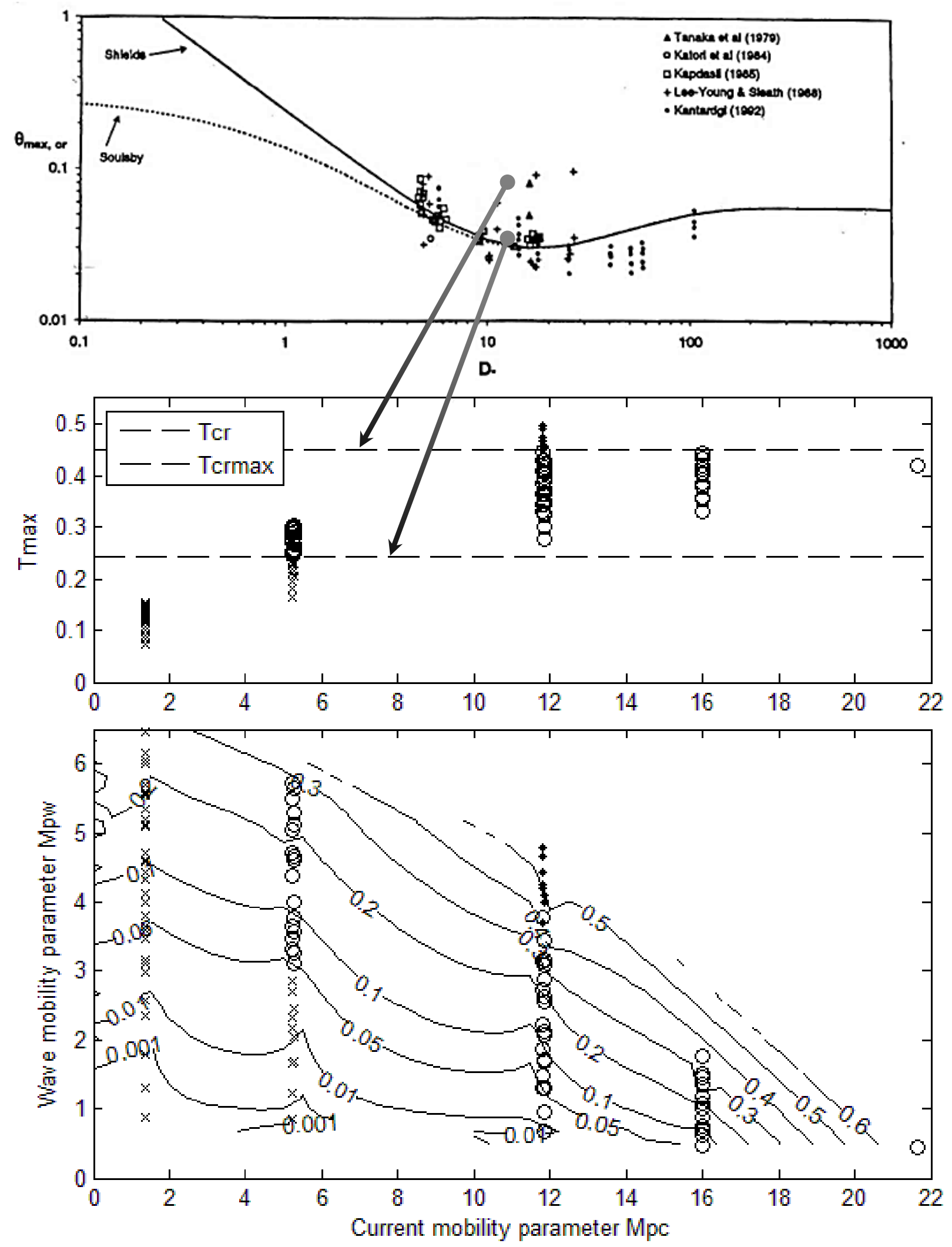

Figure 4 - Calculated maximum bed shear stress related to the observed probability of initiation of sand motion. The top figure is the Shields diagram for combined waves and current, taken from Soulsby and Whitehouse (1997). The middle figure represents the calculated maximum bed shear stress vs. the current mobility parameter, using the Tanaka and Thu (1994) method. The horizontal lines represent the two critical shear stresses deduced from the Shields parameters observed in the above Shields diagram. Here the Shields parameter is defined as $\theta_{\max , c r}=\theta_{c r}=T_{c r} / \rho g(s-1) d_{50}$ and $D_{*}$ is the non-dimensional grain diameter given by Soulsby (1997, Eq. 75). The map in the lower figure is the same as Fig. 3, except that the symbol code used in the middle figure has been applied to each test in the lower figure. 
by the Shields curve and the maximum critical shear stress taking into account the scatter observed in the Shields diagram. These two thresholds are plotted as horizontal lines in the middle figure, and three different symbols are used to represent the three ranges of the maximum bed shear stress; the diamonds represent the data above the maximum critical shear stress found by the maximum scatter in the Shields diagram; the crosses represent the data below the critical shear stress determined by the Shields curve; and the circles represent the data between these two thresholds. From the lower part of this figure it is observed that when the maximum bed shear stress exceeds the critical shear stress, there is hardly movement of the sand (1 to $5 \%$ of the waves), but when the maximum bed shear stress exceeds the maximum critical shear stress, about $40-50 \%$ of the waves initiate the sand motion. However, the maximum critical shear stress is not reached for high values of $\mathrm{Mp}_{\mathrm{C}}$, but yet more than $50-60 \%$ of the waves move the sand at the bed. A similar observation is made for the lowest values of $\mathrm{Mp}_{\mathrm{C}}$.

Although the method of Tanaka and Thu (1994) is valid regardless of the flow regime, these results suggest that this approach tend to under-predict the observed values of the maximum bed shear stress for weakly combined flows, i.e. dominated by waves or current.

\section{Critical shear stress and predicted maximum shear stress, Soulsby and Clarke (2005)}

Soulsby and Clarke (2005) presented a model for the bed shear stress under combined random wave and currents over smooth and rough beds. Their algorithm is first defining the appropriate flow regime based on the flow parameters, and then proposes new equations for deriving the shear stresses under combined waves and current. The details of the calibration process and the algorithm can be found in their report. The data obtained from this model are $\tau_{\mathrm{m}}$ (mean bed shear stress), $\tau_{\mathrm{rms}}$, and $\tau_{\mathrm{max}}$.
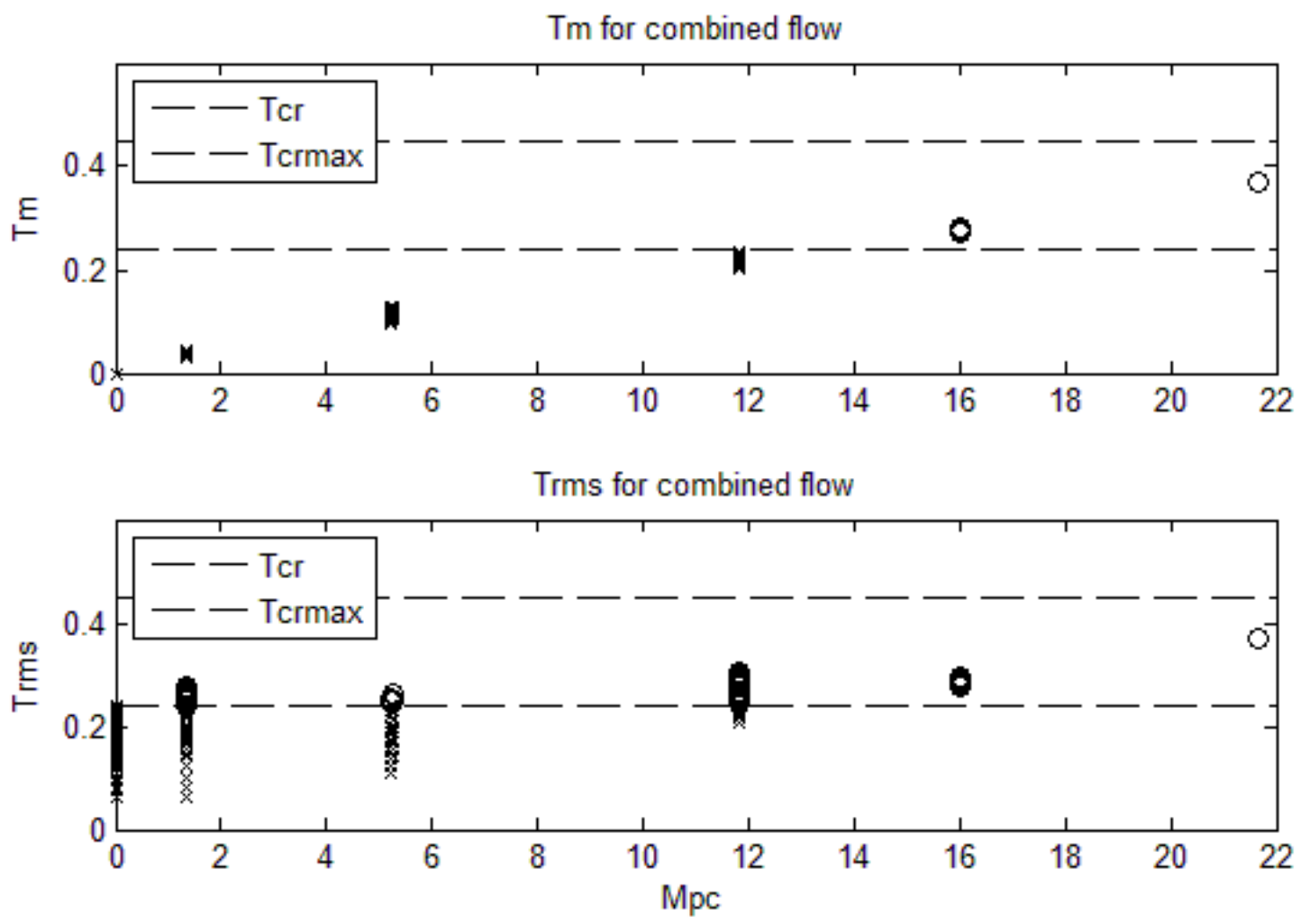

Tmax for combined flow

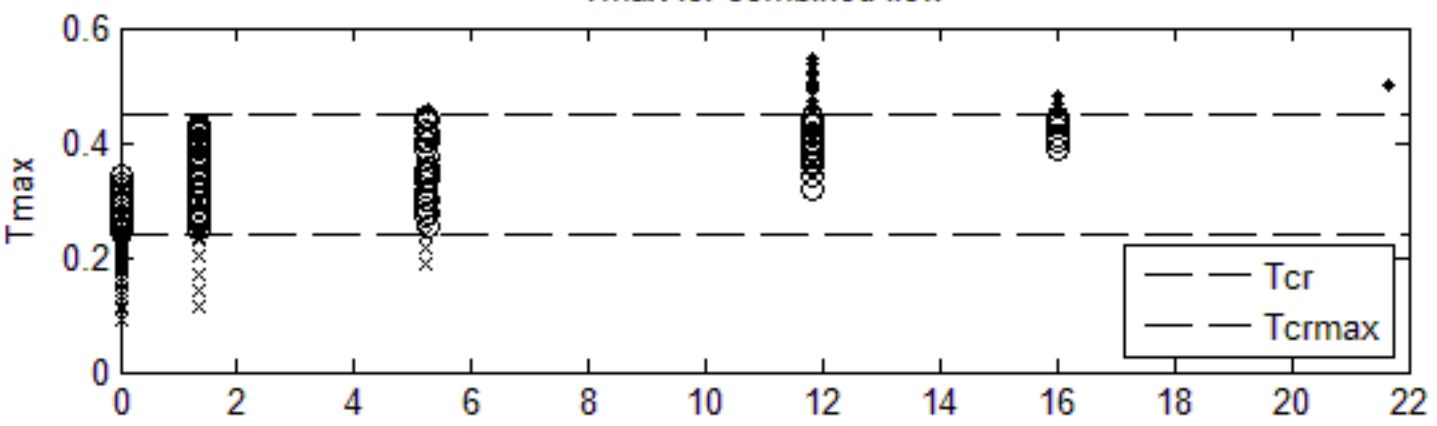

Figure 5 - Calculated mean, rms and maximum bed shear stress following the method by Soulsby and Clarke (2005), plotted against the current mobility parameter $\mathrm{Mp}_{\mathrm{c}}$. The symbol coding system is the same as presented in Fig. 4. 


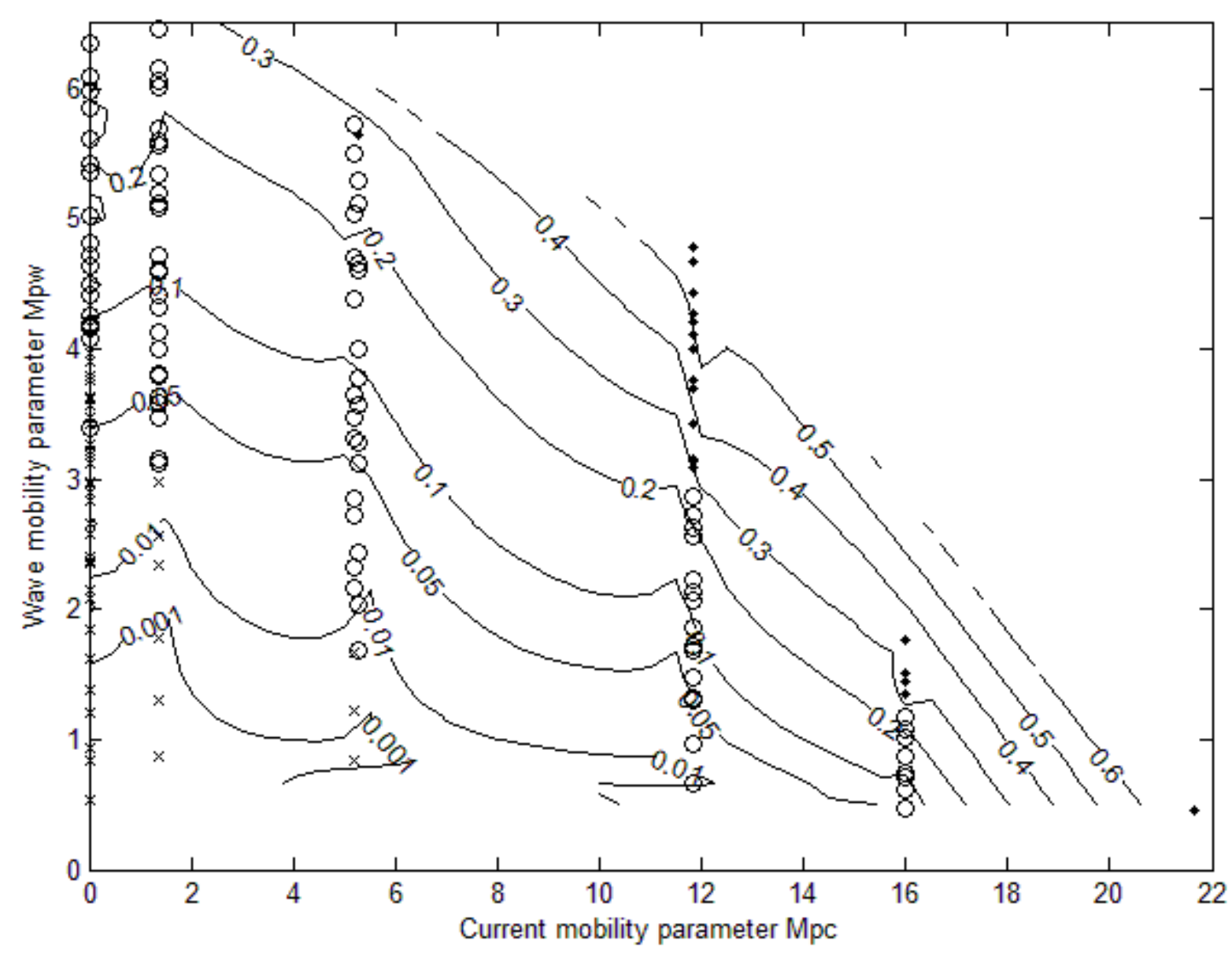

Figure 6 - Calculated maximum bed shear stress related to the observed probability of initiation of sand motion according to Soulsby and Clarke (2005); the same symbols as in Fig. 5, lower figure.

Figs. 5 and 6 present the data in the same way as in Fig. 4. The crosses correspond to a shear stress under the critical shear stress; the diamonds correspond to a shear stress above the maximum critical shear stress as deduced from the Shields diagram; the circles are the shear stresses between these two thresholds.

Fig. 6 compares the computed maximum shear stress according to Soulsby and Clarke (2005) with the observed probability to initiate the sand motion. Overall, it appears that the combined wave-current test cases for which $1 \%$ of the waves move the upper sand layer, the maximum bed shear stress is equal to the critical shear stress. Similarly, when $\tau_{\max }$ is equal to $\tau_{\text {cr } \max }$, approximately $30 \%$ of the waves initiate the motion of the sand at the bottom.

Thus it appears that the Soulsby and Clarke (2005) method agrees better with the observations than the Tanaka and Thu (1994) method. Consequently, these results suggest that the Soulsby and Clarke (2005) method can be used to describe the non-linear interaction between random waves and current.

\section{SUMMARY AND CONCLUSIONS}

1. Based on visual observations a map of the probability of exceeding the critical shear stress for the initiation of sand motion has been given by interpolating over different wave-current combinations.

2. The Tanaka and Thu (1994) method and the Soulsby and Clarke (2005) method have been used to predict the maximum bed shear stress. It appears that the Soulsby and Clarke method agrees better with the observations than the Tanaka and Thu method. Consequently, the results suggest that the Soulsby and Clarke method can be used to describe the non-linear interaction between random waves and current. 


\section{REFERENCES}

Henry, P.-Y. 2011. Seabed stability: Shear stress under random waves plus current. Tests on a flat sand layer. Master thesis, Norwegian University of Science and Technology, Department of Civil and Transport Engineering, February 2011.

Myrhaug, D. and L.E. Holmedal. 2011. Bottom friction and erosion beneath long-crested and short-crested nonlinear random waves. Ocean Engineering, 38, 2015-2022.

Shvidchenko A.B., G. Pender and T.B. Hoey. 2001. Critical shear stress for incipient motion of sand/gravel streambeds. Water Resources Research, 37, 2273-2283.

Soulsby, R. L. (1997). Dynamics of marine sands, Thomas Telford, London, 249 pp.

Soulsby, R.L. and R.J.S. Whitehouse. 1997. Threshold of sediment motion in Coastal Environments. Proc. Combined Australian Coastal Engineering and Port Conference, EA, 149-154.

Soulsby, R.L. and S. Clarke. 2005. Bed Shear-stresses Under Combined Waves and Currents on Smooth and Rough Beds, HR Wallingford, Report TR137.

Tanaka, H. and A. Thu. 1994. Full-range equation of friction coefficient and phase difference in a wave-current boundary layer, Coastal Engineering, 22, 237-254.

Wilcock, P. R. 1993. Critical shear stress of natural sediments, J . Hydraul.Eng., 119(4), 491-505. 